\title{
EDITORIAL
}

\section{Videomicrometry of lung slices: a glimpse of the dynamic airway-parenchyma interaction in emphysematous lungs}

\author{
C.Y. Seow
}

E mphysematous and fibrotic remodelling of lung tissue associated with chronic obstructive pulmonary disease (COPD) is known to alter the mechanical properties of the parenchyma and the airways, as well as the mechanical interdependence between them. This is thought to contribute to increased airway resistance in COPD [1]. Due to its dynamic nature, attempts to directly characterise the in vivo airwayparenchyma interaction have yet to produce satisfactory results. Although some airways can be visualised (and their lumen areas quantified) in vivo with high-resolution computed tomography (HRCT) [2, 3], the time resolution of HRCT is inadequate to capture rapid events associated with the airwayparenchyma interaction in the dynamic lung environment. In addition, the spatial resolution of HRCT precludes it from imaging sub-millimeter airways. In vitro studies of airway segments [4] and parenchymal strips [5, 6] suffer from their inability to reproduce the in vivo mechanical interdependence. With high temporal and spatial resolution, videomicrometry of "live" lung slices could finally allow us to examine the dynamic airway-parenchyma interaction under conditions close to that of the native lung environment. Earlier use of videomicrometry was confined to relatively static conditions, in which airway diameters were measured under steady or semi-steady states [7-9]. BERGNER and SANDERSON [10] pioneered the technique of videomicrometry with sufficient temporal and spatial resolution to describe the time courses of calcium transients and airway smooth muscle contraction in lung slices. KHAN et al. [11] have extended the technique to characterise the kinetics of bronchoconstriction in a mouse model of elastase-induced emphysema. Creative uses of this innovative technique are likely to lead to fruitful research involving analysis of complex mechanical interactions among tissue components.

As reported by KHAN et al. [11], overnight incubation of thin slices of mouse lung with elastase produced visible tissue destruction, as evidenced by patchy parenchyma and epithelial degeneration. As expected, airways in the elastase-treated slices narrowed at a greater rate and to a greater extent

STATEMENT OF INTEREST: None declared.

CORRESPONDENCE: C.Y. Seow, Dept of Pathology and Laboratory Medicine, University of British Columbia, iCAPTURE Centre/St. Paul's Hospital, Vancouver, BC, V6Z 1Y6, Canada. Fax: 1 6048069274. E-mail: cseow@mrl.ubc.ca compared with normal controls. This is likely to be due to reduced elastic loads on airway smooth muscle because of the disrupted parenchymal tethering on the airways. This can also explain the slowing of airway re-expansion that was observed upon removal of the stimulus (acetylcholine) [11].

Interestingly and somewhat unexpectedly, the cholinergic responsiveness of the airways was reduced in lung slices from in vivo elastase-treated mice (i.e. one intranasal exposure to porcine pancreatic elastase followed by 2 weeks of recovery). In mice treated with a high dose of elastase the cholinergic response was abolished [11]. This happened in spite of the presence of visible parenchymal destruction by the in vivo elastase treatment [11]. As discussed by KHAN et al. [11], several possibilities could account for this paradoxical observation. During the 2 weeks following elastase instillation, the inflammatory and reparative process might result in fibrotic remodelling of the airways, which could increase airway stiffness and thus resistance to shortening of the airway smooth muscle. The same process could also lead to a loss of contractile phenotype in the airway smooth muscle. Experimental bias might also favour the selection of fibrotic airways versus those in the region of the lung slice showing most emphysematous changes. Whatever the explanation, without the innovative technique of videomicrometry these observations would not have been made. KHAN et al. [11] have demonstrated that reliance on histological analysis of lung morphology or biochemical analysis of protein composition in lung tissues could lead to erroneous conclusions regarding changes in lung function and airway responsiveness.

Undoubtedly, videomicrometry of lung slices will prove to be a useful tool for studying the dynamic interaction of airways and parenchyma. However, there are limitations to this technique. The native environment of the lung is not exactly reproduced in the lung slice. Notably, the absence in the lung slice of the perpetual volume changes of an in vivo lung was associated with the continuous action of tidal breathing and occasional deep inspirations. Another possible difference of lung slice preparation from an intact lung is hinted at by the absence of large folds in the epithelial layer of constricted airways in the lung slice. Anyone familiar with lung histology will know that airway constriction is associated with a folded epithelial layer and basement membrane. One proposed mechanism regarding the formation of these folds requires an intact basement membrane along the airway tree [12] 
According to the hypothesis, constriction of the airway reduces the space between the muscle layer and the basement membrane. As the lamina propria-submucosa, the deformable material between the muscle layer and the basement membrane, is incompressible, folding (or bulging) of the basement membrane occurs because of the increased pressure in the lamina propria-submucosa layer, and it protrudes into the lumen space as the airway constricts. As there are collagenelastin fibres tethered between the muscle layer and the basement membrane at semi-regular intervals [12], the familiar folds of the basement membrane (and the epithelial layer) appear in constricted airways. If this hypothesis is correct, folding of the basement membrane should not occur in airways embedded in a lung slice, because pressure cannot be contained in the lamina propria-submucosa layer when airways are sliced. Therefore, the resistance to airway narrowing stemming from folding of the submucosa and the basement membrane layer $[12,13]$ will be absent in the thin slice of airways studied using videomicrometry.

Despite the imperfect preservation of an in vivo lung environment, lung slices do possess some degree of intact parenchymal tethering with the airways. With its high temporal and spatial resolution, videomicrometry can capture the strain history of the parenchymal tethers as the airways constrict. With known mechanical properties of the lung parenchyma [14], a mathematical model using finite element analysis could be employed to work out the exact stress-strain relationship of airways and other lung components, including other airways. The use of videomicrometry to study mechanical properties of biological tissue is still in its infancy. KHAN et al. [11] have offered us a glimpse of what this technique can do in terms of revealing changes in tissue properties due to disease processes.

\section{REFERENCES}

1 Saetta M, Turato G, Maestrelli P, Mapp CE, Fabbri LM. Cellular and structural bases of chronic obstructive pulmonary disease. Am J Respir Crit Care Med 2001; 163: 1304-1309.
2 Brown RH, Scichilone N, Mudge B, Diemer FB, Permutt S, Togias A. High-resolution computed tomography evaluation of airway distensibility and the effects of lung inflation on airway caliber in healthy subjects and individuals with asthma. Am J Respir Crit Care Med 2001; 163: 994-1001.

3 Brown RH, Mitzner W. Airway response to deep inspiration: role of nitric oxide. Eur Respir J 2003; 22: 57-61.

4 Gray PR, Mitchell HW. Intramural elastase injection increases responsiveness of isolated bronchial segments. Pulm Pharmacol 1996; 9: 239-243.

5 Tanaka R, Ludwig MS. Changes in viscoelastic properties of rat lung parenchymal strips with maturation. J Appl Physiol 1999; 87: 2081-2089.

6 Ito S, Ingenito EP, Brewer KK, et al. Mechanics, nonlinearity, and failure strength of lung tissue in a mouse model of emphysema: possible role of collagen remodeling. J Appl Physiol 2005; 98: 503-511.

7 Dandurand RJ, Wang CG, Phillips NC, Eidelman DH. Responsiveness of individual airways to methacholine in adult rat lung explants. J Appl Physiol 1993; 75: 364-372.

8 Martin C, Uhlig S, Ullrich V. Videomicroscopy of methacholine-induced contraction of individual airways in precision-cut lung slices. Eur Respir J 1996; 9: 2479-2487.

9 Kott KS, Pinkerton KE, Bric JM, Plopper CG, Avadhanam KP, Joad JP. Methacholine responsiveness of proximal and distal airways of monkeys and rats using videomicrometry. J Appl Physiol 2002; 92: 989-996.

10 Bergner A, Sanderson MJ. Acetylcholine-induced calcium signaling and contraction of airway smooth muscle cells in lung slices. J Gen Physiol 2002; 119: 187-198.

11 Khan MA, Kianpour S, Stämpfli MR, Janssen LJ. Kinetics of in vitro bronchoconstriction in an elastolytic mouse model of emphysema. Eur Respir J 2007; 30: 691-700.

12 Seow CY, Wang L, Paré PD. Airway narrowing and internal structural constraints. J Appl Physiol 2000; 88: 527-533.

13 Lambert RK, Codd SL, Alley MR, Pack RJ. Physical determinants of bronchial mucosal folding. J Appl Physiol 1994; 77: 1206-1216.

14 Gao J, Huang W, Yen RT. Mechanical properties of human lung parenchyma. Biomed Sci Instrum 2006; 42: 172-180. 